\title{
RESPUESTA DE LA REGENERACIÓN NATURAL DE LA Uncaria guianensis (Aubl.) J.F. Gmel “UÑA DE GATO”, AL EFECTO DE LA LUZ EN BOSQUES SECUNDARIOS DENTRO DEL BOSQUE NACIONAL ALEXANDER VON HUMBOLDT, PUCALLPA - PERÚ
}

\section{THE EFFECT OF LIGHT ON THE NATURAL REGENERATION OF Uncaria guianensis (Aubl.) J.F. Gmel, "CAT'S CLAW", IN A SECONDARY FOREST AT THE "BOSQUE NACIONAL ALEXANDER VON HUMBOLDT”, PUCALLPA - PERU}

\author{
Ayling Wetzell Canales-Springett ${ }^{1}$, Aldo Ceroni Stuva ${ }^{2}$, Gilberto Domínguez Torrejón ${ }^{3}$ y Andrés Castillo \\ Quiliano $^{4}$
}

\section{Resumen}

El presente estudio de investigación fue llevado a cabo en el ámbito del Bosque Nacional Alexander Von Humboldt-BNAVH, en la jurisdicción del distrito de Irazola, Provincia de Padre Abad y los distritos de Tournavista y Puerto Inca, Provincia de Puerto Inca, Regiones de Ucayali y Huánuco respectivamente. El objetivo fue evaluar los factores climáticos, específicamente iluminación, que influyen en la regeneración natural de la Uncaria guianensis (Aubl.) J.F. Gmel., (Uña de gato) en bosques naturales secundarios, ubicados dentro y fuera del BNAVH. La evaluación, se realizó en tres tipos de Bosques Secundarios caracterizados por la edad o tiempo de establecimiento de los mismos, dentro de los cuales se establecieron parcelas semipermanentes de evaluación. En cada una de ellas se midieron variables biológicas de la regeneración natural de la $U$. guianensis respecto al factor iluminación que llega al sotobosque. Se encontró, dependiendo de la edad del bosque, un promedio de más de 1000 individuos de regeneración natural por ha., en buenas condiciones fitosanitarias, y con diámetros promedio de $13 \mathrm{~mm}$. Respecto a la altura se observó que, el número total de sus individuos de 30 a $90 \mathrm{~cm}$ de altura era de 160 , el de los individuos de 90 a $150 \mathrm{~cm}$ de altura era de 160 , el de los individuos de 150 a $210 \mathrm{~cm}$ de altura era de 220 , y el de sus individuos de más de $2 \mathrm{~m}$ de altura era de 610 . El porcentaje de iluminación promedio encontrado en los Bosques evaluados, fue muy similar, aunque supera casi en un $6 \%$ a la cantidad de luz que llega al sotobosque de la selva tropical según lo indican los autores. Para evaluar la cantidad de iluminación promedio que llega al sotobosque, se tomaron datos en 12 parcelas de bosque secundario de 3 años de edad, en 6 parcelas de bosque secundario de 6 años de edad y en 6 parcelas de bosque secundario de más de 8 años de edad, ya que, cuanto más jóvenes fueran estos bosques, mayor sería el porcentaje de iluminación que llega al sotobosque.

Palabras clave: Bosque Nacional, iluminación, Uncaria guianensis (Aubl.) J.F. Gmel., uña de gato, regeneración natural.

\begin{abstract}
The following research was carried out within the area around the Alexander Von Humboldt National Forest BNAVH, belonging to the districts of Irazola, Tournavista and Puerto Inca in the provinces of Padre Abad and Puerto Inca at Huanuco and Ucayali regions respectively. The aim was to evaluate how climatic factors, specifically light, influence the natural regeneration of Uncaria guianensis (Aubl.) J.F. Gmel., (Cat's Claw) in natural secondary forests, located inside and outside of the BNAVH. The evaluation was conducted in three types of secondary forest characterized by age or time of establishment thereof, within which semi-permanent plots were established for evaluation. In each one, Uncaria biological variables were measured in respect to how much light reached the understory. We observed that in these forest types more than 1000 individuals of natural regeneration in good phytosanitary conditions can be found per ha, with average diameters of $13 \mathrm{~mm}$. Respect to height, we found 160 individuals ranging from 30 to 90 $\mathrm{cm}$; 160 ranging from 90 to $150 \mathrm{~cm}$; 220 ranging from 150 to $210 \mathrm{~cm}$ and 610 ranging over $2 \mathrm{~m}$. The average light percentage found in these forests is very similar, but exceeds by almost $6 \%$ the value reported by other authors for rainforests. To evaluate the average light percenjage reaching the understory, data were taken in plots located within secondary forest with three different kinds of age or successional stage; one of three years old, another of six years and another with over
\end{abstract}


eight years. These forests were classified by age because the younger had higher percentages of light $(\%)$ in the understory.

Key words National Forest, lighting, Uncaria guianensis (Aubl.) J.F. Gmel., cat's claw, natural regeneration.

\section{Introducción.}

El conocimiento de los bosques amazónicos y sus alternativas de producción han sido durante muchos años, producto del esfuerzo de las investigaciones que han derivado en propuestas de desarrollo con enfoques conservacionistas y expansionistas (López et al., 2006).

El estudio de la estructura de los bosques amazónicos es importante en las investigaciones silviculturales porque permite efectuar deducciones importantes del origen, dinamismo y tendencias del futuro desarrollo de las comunidades forestales; ofrecen datos sobre las condiciones de hábitat y su influencia formativa de los árboles del trópico y son bases importantes para poder delinear las técnicas silviculturales a aplicar (Lamprecht, 1990). Dentro de los bosques, tropicales es posible encontrar capas o estratos de vegetación, los cuales son más conspicuos en bosques inalterados. Por tal motivo la cantidad de luz que puede llegar al suelo varía. En algunos casos la estratificación suele estar poco definida, ya que el plan de crecimiento de muchas especies puede ocupar más de un estrato a la vez, como en el caso de las plantas epífitas, lianas y extranguladoras (Golley, 1983). En un bosque lluvioso tropical denso, por lo general, solo hasta un $2 \%$ de la luz que llega al dosel superior, atraviesa por completo la densa vegetación. En áreas de bosque más abierto, bosques intervenidos por ejemplo, la luz puede filtrarse más fácilmente (Chadzon \& Pearcy, 1991). Otros autores señalan que, la estratificación de la vegetación en los bosques tropicales lluviosos, influye sobre el microclima interior del bosque. Señalan que cerca de un $6 \%$ de la luz que alcanza el suelo lo hace directamente en forma de salpicaduras de rayos solares, gracias a ventanas abiertas en la bóveda del dosel superior, mientras que un $44 \%$ proviene de la luz reflejada y transmitida (Denslow, 1987; Golley, 1983; Lamprech, 1990). En los bosques tropicales lluviosos y montanos de la selva peruana, podemos encontrar diferentes niveles de iluminación y temperatura, lo cual contribuye a su alta diversidad. El Perú es uno de los diez países megadiversos" del mundo, pues contiene aproximadamente el $70 \%$ de la diversidad biológica del planeta, 84 zonas de vida y 17 zonas transicionales de las 104 existentes, así como 28 tipos de climas de los 32 existentes en el planeta (Brack, 2000; Mercado, 2010). Dentro de la diversidad de plantas medicinales que posee la Amazonía peruana, está la "uña de gato". Las dos especies más conocidas pertenecen al género Uncaria. La característica que le da el nombre común a estas especies son las espinas en forma de uñas que tienen en sus tallos. La Uncaria tomentosa (Willd.) D.C tiene hábito trepador, es larga y tiene espinas semicurvas que le facilitan trepar por los troncos de los árboles. La U. guianensis (Aubl.) J.F. Gmel., tiene hábito rastrero $\mathrm{y}$ es más pequeña y sus espinas nítidamente curvas, como cuernos de cabra, le dificulta la adhesión a otras plantas (Melo, 2012).

El conocimiento de este valioso recurso ha sido preservado por tradición oral de generación en generación, llegando sólo a trascender el campo de la medicina occidental en las últimas décadas (Flores, 1995; Domínguez, 1997; Zavala \& Zevallos, 1996; Quinteros, 2001; Quevedo \& Gil, 1998). Hoy día, esta especie se usa en toda la Amazonia y tiene un mercado nacional e internacional cada vez mayor, debido a que aproximadamente el $80 \%$ de la población mundial recurre a la medicina tradicional para atender sus necesidades primarias médicas, arriesgando su abastecimiento futuro (Gómez, 2004; Melo, E. 2012).

La U. guianensis, presenta algunas características distintas con respecto a la $U$. tomentosa. Por ejemplo algunos autores señalan que esta especie prefiere para crecer bosques secundarios, antes que otro tipo de ecosistema. También señalan que su etapa reproductiva se da unos meses antes que la $U$. tomentosa. La floración de la $U$. guianensis se da de febrero a junio y fructifica de abril a agosto (Melo, 2012). Sin embargo, también se le puede encontrar en bosques ligeramente intervenidos, donde se encuentran árboles muy desarrollados y con grandes diámetros, posiblemente muy viejos, donde sólo hubo extracción selectiva de especies maderables comerciales (Lombardi \& Zevallos, 1999). Por medio de un estudio sobre las dos especies en el Estado de Acre (Brasil) se determinó que la uña de gato, la especie U. guianensis (Aubl.) J.F. Gmel., crece con mayor frecuencia en ecosistemas de várzea (33 plantas/ha) y en el bosque secundario (11 plantas/ha) y no tanto en ecosistemas de tierra firme $(1,7$ plantas/ha). Aparentemente, ambas especies prefieren diferentes tipos de hábitats. La trepadora U. tomentosa prefiere el monte cerrado o los bosques poco intervenidos $\mathrm{y}$ generalmente crece en densidades bajas; en cambio la $U$. guianensis crece mejor en el bosque secundario, a orillas de ríos o carreteras donde puede crecer en grandes concentraciones (Melo, 2012). En estos ambientes la entrada de rayos solares hasta la superficie del suelo es escasa. Sin embargo, esta circunstancia parece ser aprovechada por las semillas de esta especie para germinar, estableciendo pequeños rodales de regeneración natural (Lombardi \& Zevallos, 1999). Los autores también reportan que 
puede ser hallada junto con los siguientes árboles: huayruro (Ormosia sp.), marupá (Simarouba amara), lupuna (Chorisia sp.), shihuahaco (Dypteris alata), cumala amarilla (Iriartea sp.), banderilla roja (Jacaranda sp.), cedro (Cedrela odorata), caoba (Swietenia macrophylla), roble amarillo (Terminalia tarapotensis), tornillo (Cedrelinga catenaeformis), capirona (Calycophyllum spruceanum), balata (Pouteria sp.), zapotillo blanco (Quararibea sp.), entre las más importantes (Lombardi \& Zevallos, 1999).

El objetivo principal de este trabajo fue estudiar condiciones del hábitat natural de la especie, evaluando para ello un factor ambiental en especial, la iluminación y, cómo influye en el desarrollo de la regeneración natural de la Uncaria guianensis (Aubl.) J.F. Gmel (Uña de gato), a fin de facilitar su manejo en bosques naturales para lograr un adecuado uso sostenible del recurso.

\section{Materiales y métodos. Área de estudio}

El presente estudio de investigación fue llevado a cabo en el Bosque Nacional Alexander Von Humboldt- BNAVH y en áreas aledañas con bosques secundarios presentes. El BNAVH se ubica entre el Km. 100 y 150 de la carretera Federico Basadre, en la jurisdicción del distrito de Irazola, Provincia de Padre Abad y los distritos de Tournavista y Puerto Inca, Provincia de Puerto Inca, Regiones de Ucayali y Huánuco, respectivamente.

El área de trabajo abarcó aproximadamente 100 ha de bosque secundario, distribuidos dentro del BNAVH, y en los límites del Bosque Nacional, así como en el kilómetro 12 de la Carretera Marginal de la Selva Norte y en el kilómetro 96 de la Carretera Federico Basadre, lugares que se muestran en la Figura 1. La Carretera Marginal, conecta el asentamiento rural "Alexander Von Humboldt" (Km. 86 de la Carretera Federico Basadre), con el centro poblado de Puerto Inca. Altitudinalmente ocupa elevaciones entre 250 a $500 \mathrm{~m}$ sobre el nivel del mar. La temperatura promedio es de $25^{\circ} \mathrm{C}$., presentando una máxima de $35^{\circ} \mathrm{C}$ y una mínima de $14^{\circ} \mathrm{C}$. Los meses más lluviosos son de octubre a marzo y los más secos de mayo a setiembre. El área donde se establecieron las parcelas de estudio, pertenecía a la Zona de Vida clasificada como Bosque muy húmedo Pre-montano Tropical (bmh-PT).

\section{Caracterizacion del área de investigación}

La fisiografía del área donde se realizó la presente investigación, estaba conformada por un paisaje colinoso, constituido por terrenos ondulados de contornos y pendientes suaves, hasta colinas bajas accidentadas, las mismas que se encontraban enmarcadas en las microcuencas de los ríos San Alejandro y Macuya, las cuales están constituidas por una red de tributarios permanentes y quebradas encajonadas. Los bosques evaluados presentaban una composición florística muy heterogénea. Las especies más abundantes que caracterizan al bosque son: catahua (Hura crepitans), copaiba (Copaeifera sp.), estoraque (Myroxilon balsamun), lupuna (Ceiba pentandra), shihuahuaco (Dipterix odorata), ana caspi (Apuleia leiocarpa), camungo moena (Vochysia sp.) y maquisapa ñaccha (Apeiba aspera). Sin embargo, los lugares en donde se trabajó, al ser áreas intervenidas, no mostraban muchos individuos de los árboles mencionados y en su mayoría estaban ubicados cerca de predios agrícolas o agroforestales, pertenecientes a las comunidades nativas, campesinas y a pobladores colonos de los asentamientos rurales de la zona.

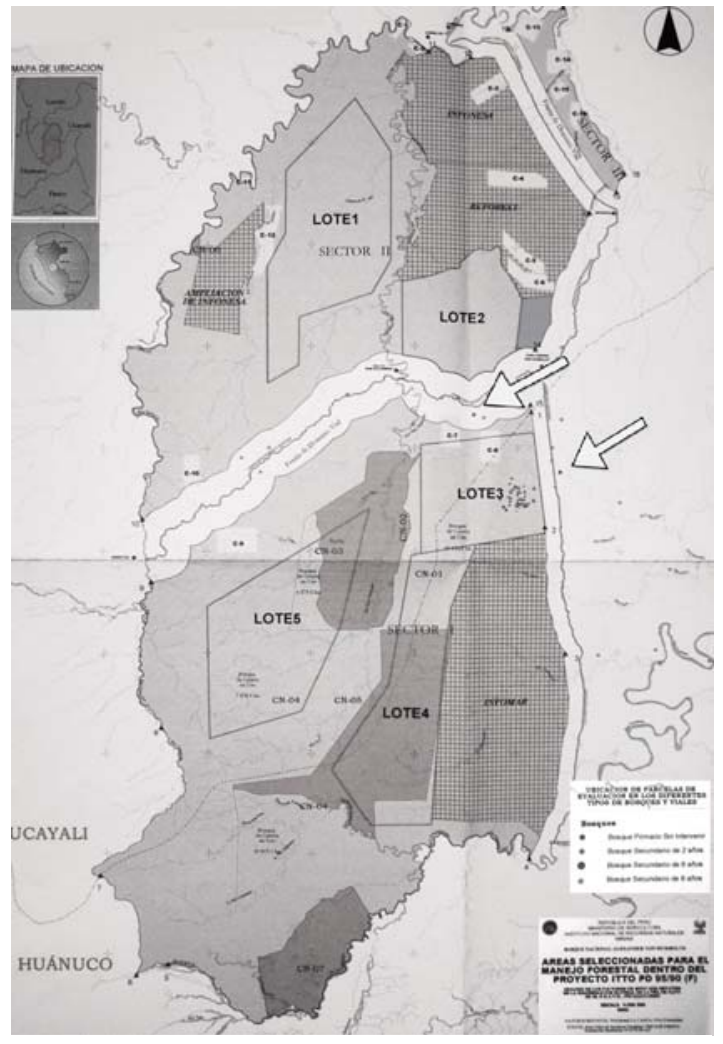

Figura 1. Mapa de ubicación del Bosque Nacional Alexander Von Humboldt y la ubicación de los Bosques Secundarios evaluados Fuente: Elaboración propia.

\section{Metodoloqía de evaluación.}

Para el presente estudio, se realizó una exploración de campo para la identificación de bosques secundarios con presencia de Uncaria guianensis (Aubl.) J.F. Gmel, tomando en cuenta la etapa o estadío sucesional (edad) de los mismos, pues por cada estadío se presentan variaciones en su composición y abundancia florística (Figura 2) y se determinaron 3 áreas de muestreo (Tabla 1). 
Dentro de los 3 tipos de bosque seleccionados (Tabla 1), se establecieron parcelas de medición de 10 x $10 \mathrm{~m}^{2}$, es decir de $100 \mathrm{~m}^{2}$ o 0.01 ha. El tamaño, número y ubicación de cada parcela siguió la Metodología para el Estudio de la Vegetación, propuesta por Matteucci et al. 1982. Una vez establecida, cada parcela fue limpiada, señalizadas, marcadas y evaluadas. Cada tipo de bosque seleccionado, contó con un número determinado de parcelas de evaluación (repeticiones). (Tabla 1)

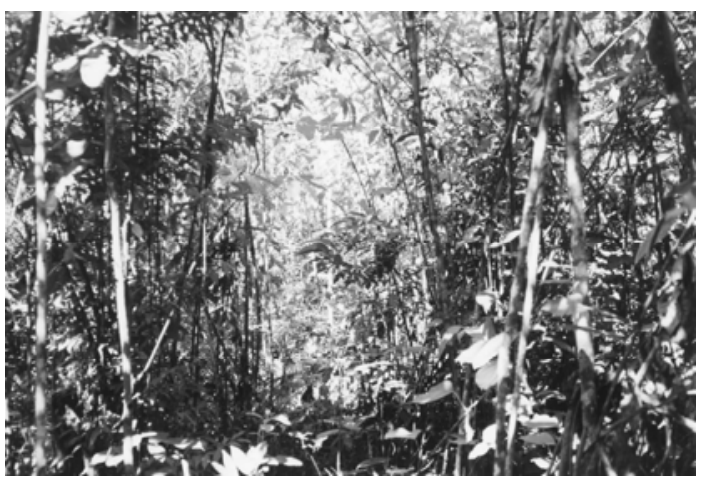

Figura 2. Trocha principal abierta para luego seleccionar la ubicación de las parcelas de evaluación dentro del Bosque Secundario de 8 años ubicado dentro del BNAVH.

En cada parcela establecida, se evaluó la regeneración natural de Uncaria guianensis (Aubl.) J.F. Gmel., que tuviera aproximadamente entre 3 meses a 2 años de edad, es decir aquellos individuos que mostraran un hábito de crecimiento más parecido al de un arbusto joven que al de una liana.

Tabla 1. Clasificación de los Bosques Secundarios evaluados de acuerdo a su estadío sucesional o periodo de apertura

\begin{tabular}{|c|c|c|c|}
\hline \multicolumn{2}{|c|}{ Bosque Secundario } & \multirow{2}{*}{$\begin{array}{l}\text { Superficie } \\
\text { total }\end{array}$} & \multirow{2}{*}{$\begin{array}{l}\text { Numero } \\
\text { de } \\
\text { Parcelas }\end{array}$} \\
\hline Tipos & Ubicación & & \\
\hline $\begin{array}{l}\text { BS de } 1 \text { a } 3 \\
\text { años de } \\
\text { descanso }\end{array}$ & $\begin{array}{l}\text { Km. } 98 \text { Carretera Federico } \\
\text { Basadre dentro de los } \\
\text { límites del BNAVH }\end{array}$ & 2 has. & 12 \\
\hline $\begin{array}{l}\text { BS de } 5 \text { a } 7 \\
\text { años de } \\
\text { descanso }\end{array}$ & $\begin{array}{l}\text { Caserío "Macuya"- Km. } 12 \\
\text { de la Carretera Marginal, } \\
\text { cerca de un área de } \\
\text { producción } \\
\text { agrosilvopastoril. }\end{array}$ & 1 ha. & 6 \\
\hline $\begin{array}{l}\text { BS de } 8 \text { a } 10 \\
\text { años de } \\
\text { descanso }\end{array}$ & $\begin{array}{l}\text { BNAVH - Altura del Km. } \\
3 \text { de la Carretera Principal } \\
\text { de acceso a la estación } \\
\text { experimental del ex - } \\
\text { proyecto ITTO. }\end{array}$ & $1 \mathrm{ha}$. & 6 \\
\hline
\end{tabular}

En la Figura 3 se observa en forma esquemática la disposición de las parcelas de evaluación dentro de los diferentes BS seleccionados.
Adicionalmente se hicieron algunas observaciones dentro de un Bosque Primario, para tener una referencia frente a los demás tipos de Bosque estudiados.

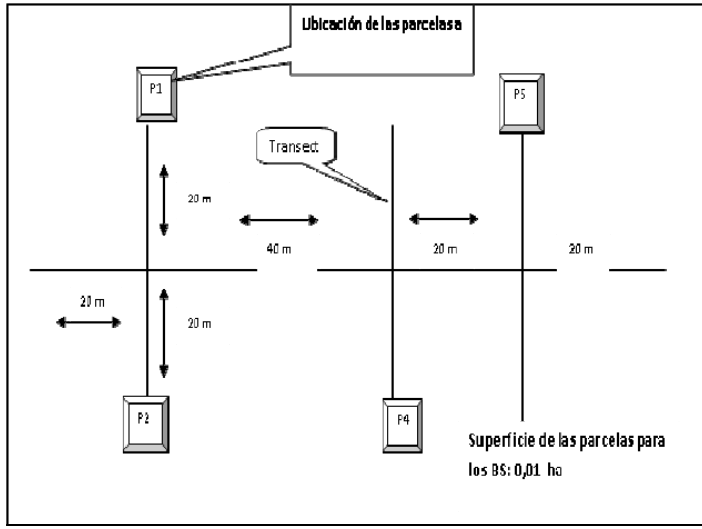

Figura 3. Ubicación y distribución usada para las parcelas de evaluación en los Bosques Secundarios evaluados.

\section{Parámetros ambientales evaluados}

Se evaluó la respuesta de la regeneración natural de la U. guianensis (Aubl.) J.F. Gmel., frente al factor ambiental iluminación, tomando en consideración que la mayoría de los autores señalan a esta especie como heliófila, aunque dentro de estos registros no se señala que se comporte como una planta heliófita en todos los estadios de su crecimiento y desarrollo. Los datos de iluminación se obtuvieron analizando los siguientes parámetros:

Intensidad de luz que llega al sotobosque: Para obtener estos datos se usaron luxímetros de lectura directa, los datos se tomaron en lux y se determinó el porcentaje de luz que llega al sotobosque, hasta la regeneración natural, con respecto a la cantidad de luz total que llega a campo abierto o al dosel superior (copas de los árboles). Se consideró que la intensidad de luz que llegaba al sotobosque, dependía de la cobertura arbórea que tuviera la parcela, según el tipo y sobre todo edad de establecimiento del bosque secundario en el que se encontraba la misma. En la Tabla 2 se registra la clasificación de los datos por rangos de iluminación.

Edad de los Bosques Secundarios: se tomó en cuenta este factor puesto que dependiendo de la edad de los bosques secundarios, la cantidad de luz que ingresa al sotobosque varía. Según experiencias de campo ya realizadas, cuanto más antiguo sea un bosque secundario tendrá más tiempo en proceso de recuperación (sucesión), la cobertura arbórea será mayor y el ingreso de luz será menor y viceversa. Se evaluó como afectan estas condiciones de cobertura y luz a la regeneración de U. guianensis (Aubl.) J.F. Gmel. 


\section{Variables biolóqicas evaluadas}

Para evaluar la influencia del factor ambiental luz o iluminación, sobre la regeneración natural de $U$. guianensis (Aubl.) J.F. Gmel, se midieron las siguientes características o variables biológicas

a. Número de individuos por parcela (Densidad por parcela). En cada parcela de $100 \mathrm{~m}^{2}$, se hizo un conteo simple de individuos vivos presentes (Figura 4). De este conteo se calculó la densidad de individuos en el área total por cada tipo de Bosque Secundario (BS).

b. Diámetro aproximado de la regeneración natural dentro de cada parcela. Para medir el diámetro promedio de cada uno de los individuos de la regeneración natural de $U$. guianensis, se usó un vernier (Figura 4). Se midieron todos los individuos hallados en todas las parcelas, a $30 \mathrm{~cm}$ de distancia del suelo.

c. Niveles de Vigor o Estado Fitosanitario de la regeneración natural de la Uña de qato dentro de cada parcela. Para determinar el vigor o estado fitosanitario de las plantas se tomaron los datos relacionados con estas características y se ordenaron por rangos de porcentajes (Tablas 3 y 4 ).

Tabla 2. Rangos de iluminación (\%) que llega hasta el sotobosque.

\begin{tabular}{ccc}
\hline $\begin{array}{c}\text { Numeración para } \\
\text { los Intervalos de } \\
\text { iluminación }\end{array}$ & $\begin{array}{c}\text { \% Iluminación } \\
\text { sotobosque }\end{array}$ & $\begin{array}{c}\text { Etiquetas por } \\
\text { valor de } \\
\text { iluminación }\end{array}$ \\
\hline 1 & $1-10 \%$ & Muy poco \\
2 & $11-20 \%$ & Poco \\
3 & $21-30 \%$ & Moderado \\
4 & $31-40 \%$ & Iluminado \\
5 & $41-50 \%$ & Bien Iluminado \\
6 & $>50 \%$ & Muy iluminado \\
\hline
\end{tabular}

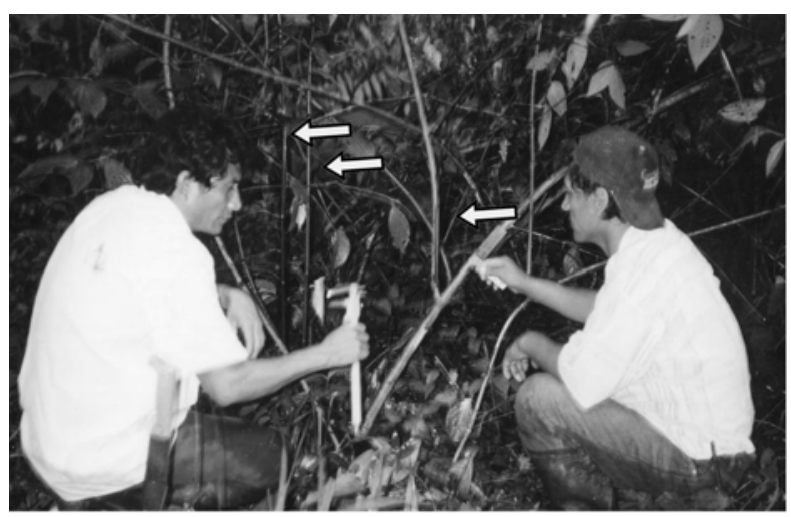

Figura 4. Medición de densidad y diámetro de la regeneración natural de Uncaria guianensis (Aubl.) J.F. Gmel.
Para poder manejar luego estas observaciones de manera cuantitativa se diseñó un sistema de valoración de vigor para cada parte observada en los individuos evaluados en el cual se ordena la información por presencia o ausencia de ramas viejas, ramas jóvenes o el estado fitosanitario de las hojas de cada uno de los individuos de $U$. guianensis que fueran hallados en cada parcela trabajada. (Tablas 3, 4 y 5).

Tabla 3. Valores de vigor o estado fitosanitario de acuerdo al porcentaje de los diferentes tipos de ramas encontradas en los individuos de la regeneración natural de Uncaria guianensis (Aubl.) J.F. Gmel., en cada parcela evaluada.

\begin{tabular}{ccc}
\hline $\begin{array}{c}\text { Ramas } \\
\text { Viejas }\end{array}$ & $\begin{array}{c}\text { Ramas } \\
\text { jóvenes }\end{array}$ & $\begin{array}{c}\text { Puntaje de } \\
\text { Vigor }\end{array}$ \\
\hline $0-34 \%$ & $>65 \%$ & 4 \\
$35-59 \%$ & $65-41 \%$ & 3 \\
$60-75 \%$ & $40-25 \%$ & 2 \\
$>75 \%$ & $<25 \%$ & 1 \\
\hline
\end{tabular}

Tabla 4. Valores de vigor o estado fitosanitario de acuerdo al porcentaje de las diferentes condiciones de las hojas de la regeneración natural de Uncaria guianensis (Aubl.) J.F. Gmel., en cada parcela evaluada.

\begin{tabular}{cccccc}
\hline $\begin{array}{c}\text { Hojas } \\
\text { jóvenes }\end{array}$ & $\begin{array}{c}\text { Hojas } \\
\text { viejas }\end{array}$ & $\begin{array}{c}\text { Hojas } \\
\text { comidas }\end{array}$ & $\begin{array}{c}\text { Hojas con } \\
\text { hongos }\end{array}$ & $\begin{array}{c}\text { Hojas con } \\
\text { Manchas de } \\
\text { Necrosado }\end{array}$ & $\begin{array}{c}\text { Puntaje } \\
\text { de Vigor }\end{array}$ \\
\hline$>70 \%$ & $<30 \%$ & $0-20 \%$ & $0-20 \%$ & $0-20 \%$ & 4 \\
$50-70 \%$ & $30-50 \%$ & $21-50 \%$ & $21-50 \%$ & $21-50 \%$ & 3 \\
$30-50 \%$ & $50-70 \%$ & $51-80 \%$ & $51-80 \%$ & $51-80 \%$ & 2 \\
$<30 \%$ & $>70 \%$ & $>80 \%$ & $>80 \%$ & $>80 \%$ & 1
\end{tabular}

De acuerdo con la Tabla 3, si un individuo de la regeneración natural de $U$. guianensis presentaba un $75 \%$ del total de ramas, en mal estado, es decir ramas marchitas o viejas, se consideraba que el estado fitosanitario o vigor de la planta, era bajo o pobre, por lo que el puntaje de vigor asignado a dicha planta sería de 1 o 2. Bajo este mismo criterio se evaluaron las hojas de la regeneración natural (Tabla 4).

Finalmente, se hizo un promedio del estado fitosanitario de cada planta de acuerdo a los valores obtenidos para sus hojas y sus ramas (Tabla 5). 


\section{d. Altura alcanzada por la regeneración natural en cada parcela (Densidad de individuos seqún su altura)}

Se midió la altura de cada individuo de cada parcela establecida dentro de los Bosques Secundarios (BS) evaluados. Los datos obtenidos en estas mediciones se agruparon por rangos (clases de altura) según la longitud que alcanzaban $(30 \mathrm{~cm}, 90 \mathrm{~cm}, 1,50$ $\mathrm{m}, 2,10 \mathrm{~m}$ y más de 2,10 m).para trabajarlos de una manera más fácil y rápida. Con dichos datos, se hicieron las pruebas estadísticas correspondientes que se presentan en los capítulos de Resultados y Discusión.

\section{Análisis Estadístico.}

Para obtener una información lo más precisa posible de como afectaba o influía el factor iluminación sobre la regeneración natural de Uncaria guianensis (Aubl.) J.F. Gmel., se sometieron los resultados de las variables biológicas y los parámetros ambientales a dos pruebas estadísticas específicas: una de correlaciones utilizando el Coeficiente de Correlación por Rangos de Spearman (Tabla 6) y otra de variancias, mediante el análisis de Kruskall-Wallis.

Tabla 6. Niveles de correlación dependiendo del coeficiente $r_{\mathrm{o}}$.

\begin{tabular}{ll}
$\begin{array}{c}\text { Valor del coeficiente } r \\
\text { (positivo o negativo) }\end{array}$ & \multicolumn{1}{c}{ Significado } \\
\hline 0,00 a 0,19 & Correlación muy débil \\
0,20 a 0,39 & Correlación débil \\
0,40 a 0,69 & Correlación moderada \\
0,70 a 0,89 & Correlación fuerte \\
0,90 a 1,00 & Correlación muy fuerte \\
\hline
\end{tabular}

\section{Resultados y discusión.}

Los resultados obtenidos corresponden a los tipos de bosque analizados con sus respectivas variantes. Se evaluaron 3 tipos de Bosques Secundarios (BS), dentro de los cuales se establecieron finalmente 24 parcelas de medición. Mención aparte merecen las parcelas evaluadas en el Bosque Primario (BP), las cuales fueron 4 en total, ubicadas dentro del lote 3 del BNAVH, en la UMF 1 del ex - proyecto ITTO PD 95/90 (F). Aunque en dichas parcelas del BP no se encontró regeneración natural de ninguna de las dos especies, se reporta el porcentaje de iluminación que llega al sotobosque. Es interesante señalar que solo se encontró regeneración natural de Uncaria guianensis (Aubl.) J.F. Gmel., en un solo tipo de Bosque Secundario, a pesar de lo que señala la literatura revisada. Aun así, se evaluaron los parámetros ambientales en los 3 tipos de bosque secundarios seleccionados. Se determinó que el porcentaje de iluminación para los tipos de bosque evaluados es similar y aunque aparentemente es poco en relación a lo que llega a campo abierto, es un poco más de lo previsto para este tipo de bosques tropicales, según lo indican los diferentes autores
Tabla 5. Valores totales y absolutos para determinar el vigor o estado fitosanitario de la regeneración natural de Uncaria guianensis (Aubl.) J.F. Gmel., en cada parcela evaluada.

\begin{tabular}{ccr}
\hline Estado Fitosanitario & $\begin{array}{c}\text { Valores } \\
\text { Absolutos }\end{array}$ & $\begin{array}{r}\text { Rangos } \\
\text { de Valores }\end{array}$ \\
\hline $\begin{array}{c}\text { Vigoroso } \\
\text { Medianamente } \\
\text { vigoroso } \\
\text { Poco vigoroso }\end{array}$ & 4 & $32-25$ \\
$\begin{array}{c}\text { Enfermo/ Seco } \\
\text { Muriéndose }\end{array}$ & 2 & $24-17$ \\
\hline
\end{tabular}

consultados (Tabla 7) (Smith, 2004; Young, 1991; Jensen \& Salisbury, 1997; Lamprecht, 1990). En general parece ser que la regeneración natural de $U$. guianensis, se establece y desarrolla mejor en los Bosques Secundarios de más de 5 años de edad, y no así en los Bosques Secundarios de más de 8 años de edad o de menos de 3 años de edad (purma), a pesar que las condiciones de luz o iluminación en estas últimas eran mejores o de un mayor porcentaje de ingreso de la luz con respecto al dosel superior y al campo abierto (Tabla 8).

Análisis de Correlación dentro de los Bosques Secundarios (BS)

Se observa que el diámetro de la de la regeneración natural de Uncaria guianensis (Aubl.) J.F. Gmel., guarda una moderada correlación positiva respecto al factor iluminación, aunque no es estadísticamente significativa. La densidad y el estado fitosanitario en cambio, presenta una correlación negativa y débil con respecto a la iluminación, y la

Tabla 7. Porcentaje (\%) de iluminación promedio que llega al sotobosque de acuerdo al tipo de bosque evaluado

\begin{tabular}{lcl}
\hline Tipo de Bosque evaluado & $\begin{array}{c}\text { \% Iluminación } \\
\text { sotobosque }\end{array}$ & $\begin{array}{l}\text { Tipo de } \\
\text { iluminación }\end{array}$ \\
\hline Bosque Primario & $22 \%$ & Moderada \\
Bosque Secundario de 3 años & $23 \%$ & Moderada \\
Bosque Secundario de 6 años & $21 \%$ & Moderada \\
Bosque Secundario de 8 años & $25 \%$ & Moderada \\
\hline
\end{tabular}

prueba no es estadísticamente significativa (Tabla 9).

La altura alcanzada por los individuos evaluados, presenta correlaciones moderadas a bajas, positivas y negativas respecto a la iluminación que llega al sotobosque, aunque la prueba no resulta estadísticamente significativa.

De acuerdo a éstos resultados se evidenció que el factor iluminación, influye en la densidad y desarrollo de la planta pero, bajo ciertos rangos y a determinada edad, es decir que si las condiciones de iluminación superan el rango de tolerancia de la planta el efecto puede llegar a ser negativo. Se determinó que a cierta altura y edad, la especie no depende de manera significativa de la iluminación o radiación directa que llega del dosel superior. 
Esto nos revelaría que los individuos más jóvenes de la regeneración natural de $U$. guianensis, tienden a preferir niveles bajos de radiación directa o radiación "indirecta" (reflejada), por lo que a simple vista parecen preferir ambientes del sotobosque de los bosques secundarios, poco a moderadamente iluminados para seguir desarrollándose y creciendo en altura.

Respecto a la edad de los bosques secundarios, se encontró que la regeneración natural de $U$. guianensis crece mejor en aquellos bosques que tiene 5 a 7 años de edad (tiempo de establecimiento), aunque la prueba de correlación nos da valores bajos y estadísticamente no significativos. Es posible que el nivel de iluminación de estos bosques, sea el más adecuado para el desarrollo de esta especie, junto con otros factores ambientales de humedad o suelo. Otro factor que puede estar interviniendo en la aparición de la regeneración natural de $U$. guianensis es la competencia intra $\mathrm{e}$ interespecífica presente en ambientes como las purmas o bosques secundarios jóvenes de menos de 3 años de edad. Se determinó que la variable altura, sigue el mismo patrón que el de la densidad, es decir que encontraremos individuos en mejores condiciones y de mayor tamaño, en bosques secundarios de 5 a 7 años de edad.

Resultados estadísticos obtenidos al aplicar la prueba de Kruskal-Wallis a los datos de los Bosques Secundarios (BS)

De acuerdo a los resultados obtenidos se verificó que la densidad, diámetro $\mathrm{y}$ vigor o estado fitosanitario de la regeneración natural de Uncaria guianensis (Aubl.) J.F. Gmel., es relativamente mayor en ambientes (sotobosque de bosques secundarios) con una iluminación baja a moderada (Tabla 10).

Tabla 8. Variables biológicas evaluadas de la regeneración natural de la Uncaria guianensis (Aubl.) J.F. Gmel., dentro de los Bosques Secundarios

\begin{tabular}{lccc}
$\begin{array}{c}\text { Variables biológicas de acuerdo al tipo de } \\
\text { Bosque Secundario evaluado }\end{array}$ & $\begin{array}{c}\text { Bosque } \\
\text { Secundario } \\
\text { 7-9 años }\end{array}$ & $\begin{array}{c}\text { Bosque } \\
\text { Secundario } \\
5-7 \text { años }\end{array}$ & $\begin{array}{c}\text { Bosque } \\
\text { Secundario } \\
2-4 \text { años }\end{array}$ \\
\hline Densidad por hectárea & 0 & 1150 & 0 \\
Diámetro Promedio & 0 & 13 & 0 \\
Valoración del Vigor & 0 & 24 & 0 \\
Vigor general o Estado Fitosanitario & Nulo & Mv & Nulo \\
№ de Individuos con Alturas de $30-90 \mathrm{~cm}$. & 0 & 160 & 0 \\
№ de Individuos con Alturas de $90-150 \mathrm{~cm}$. & 0 & 160 & 0 \\
№ de Individuos con Alturas de $150-210 \mathrm{~cm}$. & 0 & 220 & 0 \\
№ de Individuos con Alturas de + de $210 \mathrm{~cm}$. & 0 & 610 & 0 \\
\hline Mv = Medianamente vigoroso (Estado Fitosanitario de la regeneración natural) &
\end{tabular}

Tabla 9. Índices de correlación entre el factor Iluminación y las variables biológicas de la regeneración natural de la Uncaria guianensis (Aubl.) J.F. Gmel., en los Bosques Secundarios evaluados

\begin{tabular}{cccc}
\hline Parámetros Biológicos & Prueba correlación & $\begin{array}{c}\text { Iluminación } \\
\text { sotobosque } \\
\%\end{array}$ & $\begin{array}{c}\text { Edad del Bosque } \\
\text { Secundario }\end{array}$ \\
\hline Densidad (№ Indv/100m2) & $r_{\mathrm{o}}$ & -0.35 & 0.27 \\
Diámetro Promedio & Nivel de significancia & 0.1 & 0.2 \\
Vigor General & $r_{\mathrm{o}}$ & 0.68 & 0.0001 \\
& Nivel de significancia & 0.14 & 1 \\
№ Indv con una altura de 30-90cm. & $r_{\mathrm{o}}$ & -0.33 & -0.51 \\
& Nivel de significancia & 0.52 & 0.2 \\
№ Indv con una altura de 90-150cm. & $r_{\mathrm{o}}$ & -0.32 & 0.0001 \\
& Nivel de significancia & 0.68 & 1 \\
№ Indv con una altura de 150-210cm. & $r_{\mathrm{o}}$ & 0.63 & 0.0001 \\
& Nivel de significancia & 0.4 & 1 \\
& Nivel de significancia & 0.62 & 0.0001 \\
№ Indv con una altura de + de 210cm. & Nivel de significancia & 0.4 & 1 \\
\hline
\end{tabular}

$N=24 \quad N$ : Número total de parcelas evaluadas en el Bosque Secundario (BS) 
Tabla 10. Prueba K-S aplicada entre los parámetros medidos del factor Iluminación y las variables biológicas de la regeneración natural de la Uncaria guianensis (Aubl.) J.F. Gmel., en los Bosques Secundarios evaluados

\begin{tabular}{|c|c|c|c|c|c|c|c|c|c|}
\hline \multirow{2}{*}{\multicolumn{2}{|c|}{ Factor Ambiental Iluminación }} & \multirow{2}{*}{$\mathrm{N}$} & \multirow{2}{*}{$\begin{array}{c}\text { Densidad } \\
\left(\mathrm{N}^{\circ} \text { Indv } / 100 \mathrm{~m}^{2}\right)\end{array}$} & \multirow{2}{*}{$\begin{array}{l}\text { Diámetro en mm } \\
\text { (a } 30 \mathrm{~cm} . \text { del } \\
\text { suelo) }\end{array}$} & \multirow{2}{*}{$\begin{array}{l}\text { Vigor o Estado } \\
\text { Fitosanitario }\end{array}$} & \multicolumn{4}{|c|}{$\begin{array}{c}\text { Densidad ( } \mathrm{N}^{\circ} \text { Indv/100m2) de acuerdo a su rango de } \\
\text { Altura }\end{array}$} \\
\hline & & & & & & $\begin{array}{l}30-90 \\
\mathrm{~cm} .\end{array}$ & $\begin{array}{l}90-150 \\
\mathrm{~cm} .\end{array}$ & $\begin{array}{l}150-210 \\
\mathrm{~cm} .\end{array}$ & $>210 \mathrm{~cm}$ \\
\hline \multirow{4}{*}{$\begin{array}{c}\% \text { de } \\
\text { Iluminación }\end{array}$} & 11 a $20 \%$ & 5 & $6 \pm 10.43$ & $11 \pm 2$ & $4 \pm 0.58$ & 3 & $4 \pm 3.54$ & $5 \pm 4.95$ & $4 \pm 4.24$ \\
\hline & 21 a $30 \%$ & 18 & $5 \pm 13.15$ & $14.83 \pm 3.19$ & $3 \pm 0.58$ & $5 \pm 3.21$ & $5 \pm 2.12$ & $4 \pm 2.52$ & $18 \pm 13.65$ \\
\hline & 31 a $40 \%$ & 1 & 0 & 0 & 0 & 0 & 0 & 0 & 0 \\
\hline & Estimado estadístico & $\mathrm{P}$ & $>0.05$ & $>0.05$ & $>0.05$ & $>0.05$ & $>0.05$ & $>0.05$ & $>0.05$ \\
\hline \multirow{4}{*}{$\begin{array}{c}\text { Edad del } \\
\text { Bosque } \\
\text { Secundario }\end{array}$} & 3 años & 5 & 0 & 0 & 0 & 0 & 0 & 0 & 0 \\
\hline & 6 años & 18 & $19 \pm 18.69$ & $12.92 \pm 3.18$ & $4 \pm 0.55$ & $4 \pm 2.71$ & $4 \pm 0.55$ & $5 \pm 3.05$ & $13 \pm 12.4$ \\
\hline & 8 años & 1 & 0 & 0 & 0 & 0 & 0 & 0 & 0 \\
\hline & Estimado estadístico & $\mathrm{P}$ & $<0.05$ & -- & -- & $>0.05$ & -- & -- & -- \\
\hline
\end{tabular}

Con relación al desarrollo en altura de la regeneración natural, se determinó que la influencia de la cantidad de iluminación que recibe no es significativa, aunque sí se pudo observar que en el sotobosque de los bosques secundarios, que presentaron una iluminación moderada, la mayoría de los individuos encontrados alcanzan alturas de más de $210 \mathrm{~cm}$ (Tabla 10). Respecto al factor Edad del bosques secundario (etapa sucesional), se encontró que solo la variable densidad de la regeneración natural de la $U$. guianensis aumenta significativamente en Bosques Secundarios de 6 años o con un rango de edad entre los 5 a 7 años (Tabla 10).

Las variables diámetro y vigor $\mathrm{o}$ estado fitosanitario de esta especie no presentan diferencias significativas en función al mismo factor evaluado.

También se encontró que la especie presenta un mejor desarrollo en altura en aquellos bosques secundarios cuya edad de establecimiento oscila entre los 5 y 7 años, observándose que su influencia es significativa en la primera etapa de crecimiento de la planta o en individuos que aún no hayan alcanzado los $30 \mathrm{~cm}$ de altura.

Esto puede deberse a que, una vez que la regeneración natural de $U$. guianensis ha germinado, su necesidad de radiación o iluminación es moderada para que logre establecerse y crezca hasta una determinada altura $(30 \mathrm{~cm}$ aproximadamente). Una vez que ha logrado establecerse, comienza a crecer y desarrollar en altura, su necesidad de iluminación (radiación) disminuye, no es muy elevada ni significativa pero, al pasar los 2 metros de altura, su demanda de iluminación aumenta.

Esto podría deberse a que en esta fase de su crecimiento su metabolismo y tasa de fotosíntesis haya cambiado y por lo tanto recién requiera de una mayor tasa de iluminación para continuar creciendo.

Todo esto implica que, esta especie necesita un ambiente moderadamente intervenido, con una iluminación también moderada a baja, pero sólo para su germinación y establecimiento inicial. Es posible 4 que la interacción del factor iluminación y edad del bosque secundario con otros factores ambientales, como la humedad, temperatura o sustrato, tenga algún efecto conjunto sobre el desarrollo de sobre las variables biológicas de la regeneración natural de esta especie.

Es importante recordar que cuanto mayor sea el diámetro de los individuos que se encuentran bajo las condiciones de estos bosques tropicales secundarios, menor será su densidad y viceversa, principalmente por el efecto generado por "la competencia inter e intraespecífica", presente en estos ecosistemas boscosos intervenidos.

\section{Conclusiones.}

1. El desarrollo de la regeneración natural de las especies de Uncaria guianensis (Aubl.) J.F. Gmel. se encuentran influenciada por la cantidad de luz que llega al sotobosque, sin ser este factor el único, ni tampoco el limitante, para que la regeneración natural de esta especie se desarrolle con éxito.

2. Aparentemente el factor iluminación es más importante para los individuos de la regeneración natural de $U$. guianensis que presentan un mayor tiempo de establecimiento o altura, respecto a los que recién se establecen o han aparecido, lo cual puede estar relacionado con su edad, cambios en sus procesos metabólicos, como su tasa de respiración y fotosíntesis, su nivel de enraizamiento y competencia con las otras especies silvestres junto a las cuales crece.

3. La regeneración natural de $U$. guianensis, tiene un fototropismo positivo marcado luego de que ya está bien establecida en campo. El porcentaje de la iluminación que reciba no necesariamente tiene que ser excesivo o abundante, ya que su crecimiento, al ser una liana, por lo general se verá orientado hacia donde exista una entrada de luz (mayor iluminación), producida por una apertura en el dosel superior del bosque.

Si los individuos de la regeneración natural de $U$. guianensis se establecen de manera silvestre en ecosistemas boscosos intervenidos antrópicamente, sin tratamientos o cuidados 
especiales y además pueden llegar a presentan un desarrollo óptimo, significaría que trabajar en su aprovechamiento y manejo sostenible no es tan complicado y podría ser considerado como una verdadera alternativa económica para la población nativa y local de la selva peruana en donde esta especie se encuentra y distribuye naturalmente.

\section{Agradecimientos.}

Los autores expresan su agradecimiento a los $\mathrm{M}$. Sc. Mercedes Flores, M. Sc. Raúl Eyzaguirre, M. Sc. Juan Torres y la Dra. Consuelo Arellano y el Dr. Carlos Reynel, docentes de la Universidad Nacional Agraria "La Molina", por su orientación en la realización de la presente investigación así como en la revisión de la información recopilada en campo y en la revisión final del documento redactado. De igual manera al INIA de Pucallpa, el ICRAF de Pucallpa, la DRAU, el INRENA de Pucallpa y Lima y a la UNU, instituciones que brindaron su apoyo técnico, logístico, y en equipos, para la toma de datos para el trabajo de investigación. De igual forma agradecer al señor Teodoro Reyes y su hijo Litson, por brindar su invalorable conocimiento tradicional del bosque, y de los trabajadores de las instalaciones del Ex Proyecto ITTO, José, Abel y Miguel, sin los cuales la realización del arduo trabajo de campo no hubiera sido posible.

\section{Literatura citada.}

Arce J. 1996. Manejo de Bosques para Producción sostenible de Uña de gato - La experiencia del Valle del Palcazú. Resúmenes del Curso de Identificación, producción, propagación y manejo de la "Uña de gato"; organizado por la Facultad de Ciencias Forestales y llevado a cabo del 13 al 15 de Noviembre de 1996, en la Universidad Nacional Agraria "La Molina".

Barriga R. 1994. Plantas útiles de la amazonía peruana; características, usos y posibilidades.. CONCYTEC. Lima-Perú. 261 Pág.

Brack A. 2000. Ecología del Perú. Editorial Bruño. LimaPerú. 495 pág.

Castillo A. 1993. Estudio de la Regeneración Natural de Cinco Especies Forestales en el Bosque Nacional Alexander Von Humboldt. Pucallpa-Perú. 1993. Tesis para optar el Grado de Magíster Scientiae. Escuela de Post-Grado, Especialidad de Manejo Forestal. Universidad Nacional Agraria "La Molina". Lima-Perú. 156 Pág.

Chadzon R.L. \& Pearcy R.W. 1991. The importance of sunflecks for forest understory plants. Bioscience 41: $760-765$.

Denslow J.S. 1987. Tropical Rainforest Gaps and Tree Species Diversity. Annual Review of Ecology and Systematics. Vol. 18: 431-451

Domínguez G. 1997. Uña de gato y producción sostenible. Publifor-Universidad Nacional Agraria "La Molina"FCF. Lima - Perú. 138 Pág.

Dourojeani M. \& Ferreyra. R. 1986. Flora y Vegetación del Perú. Enciclopedia Gran Geografía del Perú. Tomo II.
1986 Manfer-Juan Megía Baca. Barcelona - España. 323

Dourojeani M. \& Brack A. 1986. Ecología de un País Complejo. Enciclopedia Gran Geografía del Perú. Tomo II. 1986 Manfer-Juan Megía Baca. Barcelona - España. 323

Estrella E. 1995. Biodiversidad y salud en las Poblaciones Indígenas de la Amazonía. Tratado de Cooperación Amazónico - FAO - PNUD - Banco Mundial - Union Europea- MINISTERIO DE COOPERACIÓN TÉCNICA DEL REINO DE LOS PAÍSES BAJOS UNAMAZ. Secretaría Pro Tempore Quito - Ecuador. 312 Pág.

Flores Y. 1999. Estudio Experimental del Crecimiento de Uña de Gato en Plantaciones Artificiales. Programa Nacional de Investigación en Agroforestería y Cultivos Tropicales. Instituto Nacional de Investigación Agraria INIA. Pucallpa - Perú. 20 Pág.

Fowler J. \& Cohen L. 1999. Estadística Básica en ornitología. Editorial: SEO/BIRDLIFE. ISBN: 9788492190164. España.144 págs.

Golley F.B. (ed) 1983. Tropical rain forest ecosystems: Structure and function Ecosystems of the world. Amsterdam. Elsevier Scientific Pub. Co. No. 14.A. 381 Pág

Guerrero A. 2001. Lineamientos y Estructura para el Diseño de la Estrategia Nacional Forestal. Documento de Trabajo No. 9. 2001. Proyecto FAO GCP/PER/035/NET. Apoyo a la Estrategia para el Desarrollo Forestal. Lima - Perú. 142 Pág.

INADE \& APODESA.1987. Experiencias Silviculturales y de Manejo de Bosques en América Latina. Seminario Taller llevado a cabo del 3 al 21 de Agosto de 1987. Compendio de las Presentaciones. OEA - USAID JICA - COTEBE - GTZ - ACDI. Lima-Perú. 270 Pág.

Instituto Nacional de Recursos Naturales, INRENA. 1998. Proyecto ITTO PD 95/90 (F) Manejo Forestal del Bosque Nacional Alexander Von Humboldt. Plan de Manejo Forestal del Bosque Nacional Alexander Von Humboldt. INR - 86 - DGF. Lima - Perú. 98 Pág.

Instituto Nacional de Recursos Naturales, INRENA. 1996. Guía Explicativa del Mapa Forestal. INR - 49 - DGF. Lima-Perú. 131 Pág.

Instituto Nacional de Recursos Naturales, INRENA. 1995. Mapa Ecológico del Perú. Guía Explicativa y Mapa. Ministerio de Agricultura. Lima, Perú.

Jensen W. \& Salisbury F. 1997. Botánica. Segunda edición (Primera edición en español). McGRAW-HILL. México - México. 762 Pág.

Lamprecht H. 1990. Silvicultura en los Trópicos, los ecosistemas forestales en los bosques tropicales y sus especies arbóreas, posibilidades y métodos para un aprovechamiento sostenido. Instituto de Silvicultura de la Universidad de Göttingen. Cooperación TécnicaRepública Federal de Alemania. 335 Pág.

Lock O. 1994. Investigación Fitoquímica. Métodos en el estudio de productos naturales. Pontificia Universidad Católica del Perú. Fondo Editorial. Lima - Perú. 300 Pág.

Lombardi I. \& Zevallos P. 1999. Guía para el cultivo, aprovechamiento y conservación de la Uña de Gato, Uncaria tomentosa (Willd. ex Roemer \& Schultes) De Candolle. CAB - SECAB. Santa Feb de Bogotá Colombia.19 Pág. 
López A., Bicerra E. \& Díaz E. 2006. Perfil ecológico de cuatro rodales de camu camu árbol Myrciaria floribunda (H. West. ex Willd) O. Berg. en Ucayali. Revista Ecología Aplicada, Vol. 5, № 1, Lima - Perú. Pág. 4552.

Malleaux J. 1982. Inventarios Forestales en Bosques Tropicales. UNALM, OEA. Lima - Perú. 414 Pág.

Matteucci S., Colma A. \& Frangi J.L. 1982. Metodología para el Estudio de la Vegetación. CONICIT, OEA Programa Regional de Desarrollo Científico y Tecnológico. Washington, D.C. - EE.UU. 163 Pág

Mejía K. E. 1995. Rengifo. Plantas Medicinales de Uso Popular en la Amazonía Peruana. AECI-GFL-HAPIIAP. Lima-Perú. 284 Pág.

Melo M.E. 2012. 20 Frutales y plantas útiles en la vida amazónica. Centro para la Investigación Forestal Internacional CIFOR - IMAZON - FAO - PPI. Roma, Italia. ISBN 978-92-5-307007-7. 341 Pág.

Mercado W. 2010. Negocios Rurales. La economía de la biodiversidad y los bionegocios. Revista Agronegocios. Facultad de Economía y Planificación de la Universidad Nacional Agraria La Molina - UNALM. Lima, Perú. Año $4-\mathrm{N} 3$. Pág. 3-5.

Miranda E.M., Souza J.A. \& Persira R.C.A. 2001. Subsídios Técnicos para o Manejo Sustentável da unha-de-gato (Uncaria spp.) no Vale do Rio Juruá-AC. Rio Branco, Brasil. Embrapa. 21 pp. (Documentos, 68).

Nebel G., Kvist L.P., Vanclay J. \& Vidaurre H. 2000. Dinámica de los bosques de la llanura aluvial inundable de la Amazonía Peruana; efecto de las perturbaciones e implicancias para su manejo y conservación. Folia Amazónica, Vol. 11, № 1-2. Instituto de Investigaciones de la Amazonía Peruana - IIAP. Iquitos - Perú. Pág. 6597.

Nebel G. 2000. Estructura y composición florística del bosque de la llanura aluvial inundable de la Amazonía Peruana: I. El bosque alto. Folia Amazónica, Vol. 10, No 1-2. Instituto de Investigaciones de la Amazonía Peruana - IIAP. Iquitos - Perú. Pág. 91-149.

OIMT. 1999. Manual sobre la Aplicación de Criterios e Indicadores para la Ordenación sostenible de los
Bosques Tropicales Naturales. Parte A / Indicadores a Nivel Nacional. OIMT- Serie de políticas forestales $\mathrm{N}^{\circ}$ 9.1999. Yokohama. Japón. 52 Pág.

OIMT. 1999. Manual sobre la Aplicación de Criterios e Indicadores para la Ordenación sostenible de los Bosques Tropicales Naturales. Parte B / Indicadores a Nivel de la unidad de Ordenación Forestal. OIMT- Serie de políticas forestales $\mathrm{N}^{\circ} 10.1999$. Yokohama. Japón. 48 Pág.

Quevedo A. \& Gil O. 1998. Efecto de la intensidad de la luz, método de conservación y tiempo de almacenamiento en la germinación de Croton lecheri Muell. Arg. Folia Amazónica, Vol. 9, $\mathrm{N}^{\circ}$ 1-2. Instituto de Investigaciones de la Amazonía Peruana - IIAP. Iquitos - Perú. Pág. 4561.

Quinteros B. 2001. Distribución Natural y Determinación Edafoclimática de la Uncaria tomentosa (Wild.). D.C. y Uncaria guianensis (Aubl.) Gmel (Uña de gato), en la cuenca del río Aguaytía. Tesis para optar e título de Ingeniero Forestal. Universidad Nacional de Ucayali. Facultad de Ciencias Forestales. Pucallpa - Perú. 86 Pág.

Reynel C. \& Albán J. 1985. Cuatro especies forestales con potencialidad alimienticia en la amazonía peruana. Rev. For. Perú 13: 60-79

Smith R. \& Smith T. 2004 ECOLOGÍA. Cuarta Edición. PEARSON EDUCATION S.A.; Addison - Wesley. Madrid, España. 664 Pág.

Weberbauer A. 1945. El mundo vegetal de los Andes peruanos. Estudio fitogeográfico. (Nueva edición, revisada y ampliada de: Die Pflanzenwelt der peruanischen Anden, Leipzig 1911), 776 S., Lima: Ministerio de Agricultura.

Young R. \& Hurtado J. 1991. Introducción a la Ciencias Forestales. Editorial LIMUSA S. A. de C. V. México D. F. - México. 637 Pág.

Zavala C. \& Zevallos P. 1996. Taxonomía, distribución geográfica y status del Género Uncaria en el Perú - Uña de gato. INDDA-Universidad Nacional Agraria "La Molina". Lima-Perú. 106 Pág.

\footnotetext{
${ }^{1}$ Universidad Nacional Agraria La Molina, Av La Molina s/n, La Molina, Lima Perú. awetzell@lamolina.edu.pe, awetzell@gmail.com, awetzellcs@yahoo.es

2 Universidad Nacional Agraria La Molina, Av La Molina s/n, La Molina, Lima Perú. aceroni@lamolina.edu.pe

3 Universidad Nacional Agraria La Molina, Av La Molina s/n, La Molina, Lima Perú. gdominguez@lamolina.edu.pe

4 Anterior: Instituto de Investigación de la Amazonia Peruana, Pucallpa, Perú. Actual: Universidad Nacional Intercultural de la Amazonia Peruana - Yarinacocha. Pucallpa, Ucayali Perú. acastillo_2519@hotmail.com
} 